\title{
LA FIGURA E IL MINISTERO DEL DIACONO NELLA PROSPETTIVA NEOTESTAMENTARIA
}

DOI: http://dx.doi.org/10.12775/TiCz.2018.001

\section{INTRODUZIONE}

Il diaconato è in piena evoluzione. La teologia si confronta sulla sua natura e sui compiti specifici, proponendo e talvolta contrapponendo visioni diverse del diacono: intermediario tra i laici e i presbiteri, segno di Cristo servo e/o povero, "ministro della soglia", collaboratore diretto del vescovo ${ }^{1}$. La pastorale registra esperienze molto eterogenee: alcuni

* Ks. Ryszard Selejdak - dr hab. teologii, ur. 17.01.1958 r. w Kromołowie, prezbiter archidiecezji częstochowskiej, patrysta i teolog dogmatyk. Od 15 maja $1991 \mathrm{r}$. pracownik watykańskiej Kongregacji Edukacji Katolickiej. Od 1 marca 2002 r. Dyrektor Departamentu Seminariów tej Kongregacji. Od 22 kwietnia 2013 r. Dyrektor Departamentu Seminariów watykańskiej Kongregacji ds. Duchowieństwa (r.selejdak@virgilio.it).

${ }^{1}$ Cf. Comissione Teologica Internazionale, Il diaconato: evoluzione e prospettive, La Civiltà Cattolica 154 (2003) 1, p. 253-335; E. Castellucci, Il "ministero della soglia". Una lettura teologica del diaconato a partire dal Nuovo Testamento, Seminarium 48 (2008) 4, p. 615. 
diaconi si impegnano nei settori dellevangelizzazione o della carità; altri vengono impiegati prevalentemente nella liturgia e nel culto; sempre più spesso alcuni sono inviati anche stabilmente a sostituire i presbiteri in comunità che ne sono prive. Inoltre non mancano coloro che assumono responsabilità in organismi diocesani o interdiocesani. Si tratta di una gamma molto ampia di servizi che hanno il pregio di rispondere a bisogni immediati e reali, ma il limite di frenare l'individuazione di uno "specifico" del diaconato rispetto sia alla vocazione e missione laicale sia al ministero ordinato del presbitero e del vescovo.

Questarticolo intende presentare la figura e il ministero dei diaconi alla luce del Nuovo Testamento. Perciò analizzeremo i seguenti, più importanti frammenti degli scritti neotestamentari a tale riguardo: la Lettera ai Filippesi 1,1, la Prima Lettera a Timoteo 3,8-13, e gli Atti degli Apostoli 6,1-6.8-15; 7; 8,4-13. 25-40.

\section{STATUS QUAESTIONIS ESSEGETICO}

Senza presentare uno status quaestionis, delineato nel documento sul diaconato pubblicato nel 2003 dalla Commissione Teologica Interna$z^{z i o n a l e}{ }^{2}$, precisiamo la prospettiva esegetica. Ci troviamo "di fronte a una documentazione neotestamentaria scarsa, discontinua e non sempre convergente con il successivo sviluppo teologico e ministeriale del diaconato"3. È ovvio che gli esegeti odierni non possono limitarsi a riproporre gli esiti di indagini degli anni Trenta del secolo scorso, come quelle degli esegeti protestanti Wilhelm Brandt ${ }^{4}$ e Hermann Wolfgang Beyer 5 . In sostanza, tali indagini individuavano il significato fondamentale del campo semantico della diakonía nel "servizio delle mense" e, in modo più ampio, nel servizio caritativo. Sottolineando che i termini diakoneîn, diakonía e diákonos avevano un significato peculiarmente cristiano, ne coglievano in

2 Cf. Il diaconato: evoluzione e prospettive, doc. cit.

${ }^{3}$ G. Bellia, Il diaconato permanente alle origini della Chiesa, Seminarium 48 (2008) 4, p. 651.

${ }^{4}$ Cf. W. H. G. Brandt, Dienst und Dienen im Neuen Testament, Gütersloh 1931.

${ }^{5}$ Cf. H. W. Beyer, “diakonéo, diakonía, diákonos", in: Theologisches Wörterbuch zum Neuen Testament, ed. G. Kittel-G. Friedrich, vol. II, Stuttgart 1935, col. 81-93. 
maniera riduttiva gli aspetti di umile servizio del prossimo, ma finivano per oscurare qualsiasi riferimento all'autorità, che pure è implicata in un servizio ecclesiale di questo tipo ${ }^{6}$.

Certamente, va accolto l'esito principale di tali indagini lessicografiche, cioè la costatazione che nell'Antico Testamento secondo i Settanta i termini diakoneîn, diakonía e diákonos siano poco frequenti ${ }^{7}$. È perciò verosimile che anche per questo loro scarso utilizzo gli autori del Nuovo Testamento abbiano utilizzato in senso cristologico e ecclesiologico. Il verbo diakoneîn ("servire"), il sostantivo diakonía ("servizio" o "ministero") e il sostantivo diákonos, che designa un "servo" in senso generico, ma anche nel senso di "porta-parola" o "emissario". Già da indagini di questo tipo risultava la rilevanza di due passi del Nuovo Testamento, cioè Fil 1,1 e $1 \mathrm{Tm} 3,8.12$, in cui i diákonoi sono menzionati accanto gli epískopoi ${ }^{9}$.

Questa menzione dei diákonoi in Fil e in $1 \mathrm{Tm}$ diventa più significativa alla luce della Volgata e delle altre antiche versioni latine: soltanto in questi due passi neotestamentari il termine diákonos è traslitterato in latino con diaconus, mentre in tutte le altre ricorrenze è tradotto minister ${ }^{10}$. Sembra dunque che nei due passi in questione diákonos non fosse inteso dai Padri latini in senso lato - cioè "servo" -, ma in senso specifico ossia appunto "diacono".

A questo punto, si tratta di determinare l'identità e le funzioni di questi diákonoi della Chiesa delle origini. In questottica, studi come

${ }^{6}$ Cf. E. Cattaneo, I ministeri nella Chiesa antica. Testi patristici dei primi tre secoli, Milano 1997, p. 41-42.

7 Nella Settanta il verbo diakoneîn è assente, mentre il sostantivo diakonía compare per 3 volte. Piuttosto significative sono alcune delle 7 ricorrenze del sostantivo diákonos, nel senso che in Est 1,10; 2, 3; 6,3.5 il termine al plurale indica i sette eunuchi a servizio del re Assuero.

${ }^{8}$ Nel Nuovo Testamento si trovano 36 ricorrenze del verbo diakoneîn, 33 del sostantivo diakonía e 29 del sostantivo diákonos.

9 Nella Prima Lettera a Timoteo il sostantivo diákonos compare una terza volta in 4, 6, ma come titolo piuttosto generico "servo di Gesù Cristo" - attribuito a Timoteo.

${ }^{10}$ Anche in $\mathrm{Rm} \mathrm{16}$, 1, in cui la cristiana Febe è denominata "diákonos della Chiesa che è in Cencre", il sostantivo diákonos (ovviamente inteso al femminile) è tradotto "quae est in ministerio". 
quello dei biblisti Dieter Georgi ${ }^{11}$ e John Neil Collins ${ }^{12}$ hanno consentito d'integrare le acquisizioni fondamentali delle indagini degli anni Trenta del secolo scorso. In modo particolare quest'ultimo ha approfondito, alla luce della letteratura greca profana, lo studio del campo semantico della diakonía negli scritti del Nuovo Testamento e della patristica più antica. Ha messo così in luce che esso ha per significativo principale non tanto il "servizio a tavola" né, più in genere, l'umile servizio del prossimo, senza alcun esercizio d'autorità ${ }^{13}$, quanto piuttosto una serie di funzioni onorevoli di carattere intermediario sotto l'autorità di un altro ${ }^{14}$. Più esattamente - ha concluso Collins - nella Chiesa dei primi secoli "la designazione di «diacono» non deriva dal servizio a tavola, ma dal servizio a una persona. Questa persona non è il bisognoso o la comunità, ma l'epískopos, di cui il diacono è «ministro»" 15 .

\section{FONDAMENTO CRISTOLOGICO DELLA DIAKONÍA}

Per capire questa tesi è necessario soprattutto sottolineare che il fondamento neotestamentario del diacono è eminentemente cristologico. La diakonía non è altro che la dinamica di fondo della vita di Cristo: il Figlio di Dio diventato uomo, si è fatto servo degli uomini ${ }^{16}$ e, "beneficando tutti quelli che erano sotto il dominio del diavolo"17, ha rivelato loro il volto sempre e soltanto buono del Dio- $A b b \dot{a}^{18}$. A spingere Cristo a vivere da servo degli uomini è stato il desiderio di uniformarsi radicalmente alla volontà salvifica universale di Dio, anche se va precisato che Cristo

${ }^{11}$ Cf. D. Georgi, Die Gegner des Paulus im 2. Korintherbrief. Studien zur religiösen Propaganda in der Spätantike, Neukirchener-Vluyn 1964, p. 31-38.

12 Cf. J. N. Collins, Diakonía. Re-interpreting the Ancient Sources, New York-Oxford 1990.

13 Cf. ibid., p. 5-45.

${ }^{14}$ Cf. ibid., p. 193-194; 253-263; 335-337 e il successivo approfondimento in: id., A Ministry for Tomorrow's Church, Journal of Ecumenical Studies 32 (1995), p. 159-178.

15 Id., Diakonía. Re-interpreting the Ancient Sources, p. 337.

${ }_{16}$ Cf. Mt 26,28 (parallelo a Mc 10, 45); Lc 22,27.

17 At $10,38$.

18 Cf. Mc 14,36. 
ha obbedito al Padre non tanto da servo ${ }^{19}$, quanto piuttosto da Figlio ${ }^{20}$, perché ha fatto propria la volontà divina ${ }^{21}$. Comunque, per compiere la missione ricevuta dal Padre di portare tutti gli uomini dalla situazione peccaminosa in cui erano decaduti alla salvezza gloriosa dei figli di $\mathrm{Dio}^{22}$, Cristo, "non ritenne un privilegio l'essere come Dio, ma svuotò se stesso, assumendo una condizione di servo (doúlou)"23.

\section{DIMENSIONE ECCLESIOLOGICA DELLA DIAKONÍA}

Di conseguenza, la Chiesa, in quanto strumento privilegiato del Cristo Risorto per continuare la sua missione salvifica universale nella storia, si fa anchessa serva sia di Dio che degli uomini. Spinta in modo permanente dallo Spirito a conformarsi a Cristo "diacono", la Chiesa è chiamata a vivere in maniera "diaconale".

$\grave{\mathrm{E}}$ in questa dimensione diaconale della Chiesa che si collocano i ministeri ${ }^{24}$, come tutte le altre attività ecclesiali ${ }^{25}$. Animati dall'unico Spirito d'amore, tutti i cristiani partecipano, secondo la specificità dei propri ministeri o attività, allo stile di servizio che ha contraddistinto la vita di $\mathrm{Cristo}^{26}$. R. Penna sottolinea che "Viceversa, non esiste nella chiesa un ministero che possa essere compreso come «non-servizio», quasi che

19 Cf. Mt 12,18; At 4,30.

${ }^{20}$ Cf. Gv 5,19.36; 12,49; 14,31.

${ }^{21}$ Cf. Mt 26,39.42.44 (parallelo a Mc 14,36 e a Lc 22,42); Lc 2,49; Gv 4,34; 9,31; 12,49; 14,31; Eb 5,8; 10,7.9 ecc.

${ }^{22}$ Cf. ad es., Gv 6,40; Gal 1,4; Eb 2,10; Rm 8,28-30.

${ }^{23}$ Fil 2,6-7; cf. E. Castellucci, Il "ministero della soglia". Una lettura teologica del diaconato a partire dal Nuovo Testamento, art. cit., p. 617-618; H. Vorgrimler, Per una teologia del diaconato, in: Il diaconato oggi, ed. autori vari, Roma 1970, p. 45-53.

${ }^{24}$ Cf. specialmente 1 Cor 12,4-13 e anche Rm 11,3; 2 Cor 3,3.6.8; 5,18; 6,3-4; 11,23, 1 Pt 1,10-12; Eb 6,10.

${ }^{25}$ Cf. At 11,29-30; 12, 25; 19,22; Rm 15,25-30; 2 Cor 8,19-20; Gal 2,17; 2 Tm 4,5.11; Fm 13.

${ }^{26}$ Cf. specialmente 1 Cor 12,5; X. Pikaza, Il Vangelo di Marco, Roma 1996, p. 280; H. Vorgrimler, Per una teologia del diaconato, art. cit., p. 48; G. Koch-G. LanggärtnerH. Pompey, Il diaconato nella Chiesa oggi. Saggio di un approfondimento teologico del diaconato, in: Il diaconato oggi, op. cit., p. 55-63. 
il servire debba essere caratteristico di un ministero soltanto"27. In quanto battezzati in Cristo $^{28}$, i cristiani sono conformati dallo Spirito a lui ${ }^{29}$, servo di Dio e degli uomini. Essi fanno parte del corpo ecclesiale di Cristo e ne favoriscono la crescita, annunciando il vangelo e vivendo di fede, che opera mediante la carità verso il prossimo ${ }^{30}$. Concretamente esercitano varie attività o veri propri ministeri ecclesiali, tra i quali il diaconato, che è un ministero in cui si attua in modo particolarmente significativo il servizio a Dio e agli uomini.

\section{I DIACONI NELLA LETTERA AI FILIPPESI}

La prima testimonianza scritta del cristianesimo primitivo sullesistenza di diaconi, nel senso tecnico del termine, si trova nella Lettera ai Filippesi. Il prescritto della Lettera, stesa dall'apostolo Paolo probabilmente dal carcere di Efeso ${ }^{31}$ attorno al 56/5732, recita così:

Paolo e Timoteo, servi (doûloi) di Cristo Gesù, a tutti i santi in Cristo Gesù che sono a Filippi, con i vescovi e i diaconi (sýn episkópois kai diakónois).

Alla guida di questa comunità di "santi", ossia di credenti in Cri-

27 R. Penna, La diaconía nelle Lettere ai Corinzi, in: Il diaconato permanente, ed. F. Marinelli, Napoli 1983, vol. II, p. 214.

${ }^{28}$ Cf. Rm 6,3; Gal 3,27.

${ }^{29}$ Cf. Rm 8,29; Fil 3,10.

${ }^{30}$ Cf. Gal 5,6; Rm 5,25, Mt 25,31-46; 26,26-27 (parallelo a Mc 10,43-44 e a Lc 22,26-27); Lc 17,7-10.

${ }^{31}$ Cf. G. S. Duncan, St. Paul's Ephesian Ministry. A Reconstruction with Special Reference to the Ephesian Origin of the Imprisonment Epistles, London 1929; R. Fabris, Lettera ai Filippesi. Struttura, commento e attualizzazione, Bologna 2001, p. 19; J. Gnilka, Der Philipperbrief, Freiburg im Breisgau 1987², p. 19-24; W. Michaelis, Die Gefangenschaft des Paulus in Ephesus und das Itinerar des Timotheus. Untersuchungen zur Chronologie des Paulus und der Paulusbriefe, Gütersloh 1925.

32 Cf. G. Barbaglio, Alla comunità di Filippi, in: Le lettere di Paolo, ed. G. Barbaglio-R. Fabris, Roma 1990², vol. II, p. 541-542. 
sto chiamati a diventare santi ${ }^{33}$, sono posti dei "vescovi", suscitati dallo Spirito Santo per "pascere la Chiesa di Dio"34, sul modello di Cristo, "pastore supremo" 35 . Il sostantivo epískopoi ricorre qui al plurale, forse perché nella Chiesa di Filippi con questo sostantivo erano designate le guide della comunità, senza distinzione tra il vescovo locale - se ce n'era già uno solo - e i presbiteri ${ }^{36}$. Questa distinzione si sarebbe chiarificata nelle lettere d'Ignazio di Antiochia ${ }^{37}$, da cui risulta che le Chiese locali erano dirette da un unico vescovo, coadiuvato dai presbiteri, da un lato, e dai diaconi, dall'altro.

Comunque sia, la Lettera ai Filippesi menziona i diaconi subito dopo i vescovi. Il fatto stesso che Paolo non specifichi quali fossero i compiti dei diaconi è un indizio degno di rilievo perché lascia intendere che la loro configurazione ecclesiale fosse un dato di fatto già in una comunità cristiana sorta verso la metà del primo secolo. Inoltre, si può supporre che i diaconi giocassero un ruolo ecclesiale distinto da quello dei vescovi, ma anche da quello dell'apostolo Paolo e del suo collaboratore Timoteo ${ }^{38}$.

Infine, dal particolare testuale che i diaconi sono menzionati dopo i vescovi e dal significato stesso del sostantivo diákonos si evince che i diaconi, pur collaborando con i vescovi, fossero loro subordinati.

${ }^{33}$ Il Nuovo Testamento definisce "santi" tutti i cristiani in quanto, mediante il battesimo, "sono stati santificati in Cristo Gesù" e chiamati a diventare santi (cf. 1 Cor 1,2; Rm 1,7) come lui (cf. Mc 1,24; Gv 6,69), lasciandosi docilmente conformare a lui (cf. Rm 8,29; Fil 3,10-11.21) dallo Spirito Santo (cf. 2 Cor 3,18).

34 At 20,28 .

351 Pt 5,4; cf. Gv 10,11-19; Eb 13,20; Ap 7,17.

${ }^{36}$ Difatti le guide della Chiesa efesina sono dette sia "presbiteri" che "vescovi" (At 20,17.28). Tra i numerosi studiosi che propendono per questa interpretazione, ricordiamo, ad esempio, J. Lécuyer, Les diacres dans le Nouveau Testament, in: Le diacre dans l'Église et le monde d'aujourd'hui, ed. P. Winninger-Y. Congar, Paris 1966, p. 15-26.

37 Cf. S. Zardoni, I diaconi nella Chiesa. Ricerca storica e teologica sul diaconato, Bologna 1983, p. 13.

38 Peraltro Paolo e Timoteo sono accomunati dal titolo doûloi, che, pur evocando il loro servizio a Cristo, non pare coincidere con il servizio diaconale, designato con il sostantivo diákonoi. 


\section{I DIACONI NELLA PRIMA LETTERA A TIMOTEO}

La seconda menzione dei diaconi nellepistolario paolino compare in $1 \mathrm{Tm} 3,8-13$. Questo testo si concentra sulle condizioni per l'ammissione di determinati fedeli al diaconato:

Allo stesso modo [dei vescovi; cf. 3,1-7] i diaconi (diakónous) siano persone degne e sincere nel parlare, moderati nell'uso del vino e non avidi di guadagni disonesti, e conservino il mistero della fede in una coscienza pura. Perciò siano prima sottoposti a una prova e poi, se trovati irreprensibili, siano ammessi al loro servizio (diakoneitōsan, «esercitino il diaconato»). Allo stesso modo le donne siano persone degne, non maldicenti, sobrie, fedeli in tutto. I diaconi (diākonoi) siano mariti di una sola donna e capaci di guidare bene i figli e le proprie famiglie. Coloro infatti che avranno esercitato bene i loro ministero (diakonēsantes), si acquisteranno un grado degno di onore e un grande coraggio nella fede in Cristo Gesù.

Basandosi su questo testo si può puntualizzare le tre rilevanti osservazioni.

\subsection{LA PROVA DEl CANDIDATI AL DIACONATO}

Soprattutto, pare che per diventare diaconi non fosse sufficiente che un credente si mettesse a disposizione della Chiesa. Questo elenco di atteggiamenti virtuosi e doverosi costituisce una griglia essenziale di criteri per una verifica delle capacità dei candidati al diaconato, alla quale si fa cenno esplicito al v. 10: "Siano prima sottoposti a una prova (dokimazésthōsan) e poi, se trovati irreprensibili, esercitino il diaconato". I particolari di questo discernimento ecclesiale ci sfuggono: come si procedeva in concreto in questa verifica? Quanto tempo durava la prova? Chi erano gli esaminatori? Almeno per quanto riguarda quest'ultimo aspetto, possiamo ritenere più che probabile che il discernimento ecclesiale venisse portato a termine dalle guide della comunità, in modo simile a quanto la Lettera attesta che avvenne per Timoteo ${ }^{39}$. E poi verosimile che la

${ }^{39}$ Così suppone anche S. Zardoni, I diaconi nella Chiesa. Ricerca storica e teologica sul diaconato, op. cit., p. 14-15. 
prova consistesse in un periodo preparatorio, in cui i candidati, prima di essere istituiti diaconi in maniera definitiva, esercitavano - tutte o alcune - funzioni del loro ministero ${ }^{40}$. Dopodiché, erano istituiti diaconi in modo ufficiale e comunitario. Si può supporre che ciò avvenisse attraverso l'imposizione delle mani, menzionata in una circostanza simile in $1 \mathrm{Tm}$ 4, 14, che ricorda il gesto rituale dei presbiteri su Timoteo ${ }^{41}$, e in At 6, 6, che attesta lo stesso atto compiuto dagli apostoli sui sette servitori della Chiesa di Gerusalemme.

\subsection{LA COLLABORAZIONE DEI DIACONI CON IL VESCOVO}

È poi di un certo interesse il fatto che l'autore della Prima Lettera a Timoteo, con l'espressione "allo stesso modo (hōsaútōs)" $(3,8)$, passi a trattare le qualità morali e i doveri dei diaconi immediatamente dopo aver illustrato i requisiti dei vescovi. Questo dato esplicita quello già riscontrato nella Lettera ai Filippesi, in cui la figura del diacono è distinta e subordinata a quella del vescovo, anche se la vicinanza tra le due figure è tale da lasciare intuire che il diaconato fosse ritenuto come un ministero dello stesso tipo dell'episcopato.

Ma mentre la Lettera ai Filippesi menziona al plurale sia i diaconi che i vescovi, la Prima Lettera a Timoteo designa i diaconi al plurale e il vescovo al singolare. Se ne potrebbe evincere che probabilmente nella Chiesa efesina vivesse più di un diacono, mentre il vescovo locale sarebbe soltanto uno. Tuttavia diversi studiosi lo escludono, ritenendo che, sul fine del primo secolo, non si fosse ancora sviluppato il modello dellepiscopato "monarchico". Lo proverebbe la contemporanea Lettera a Tito, in cui i sostantivi presbýteroi (al plurale, 1,5) e epískopos (al singolare, 1,7 ) sono usati come sinonimi ${ }^{42}$. Comunque sia, in questa collaborazione subordinata dei diaconi alle guide della Chiesa si trova il primo elemento di specificità della loro figura.

\footnotetext{
${ }^{40}$ Cf. J. Lécuyer, Les diacres dans le Nouveau Testament, art. cit., p. 22.

${ }^{41}$ Cf. ibidem, p. 23.

${ }^{42}$ Ad esempio, lo esclude J. Lécuyer, Les diacres dans le Nouveau Testament, art.
} cit., p. 19-20. 


\subsection{LE QUALITÀ MORALI E I DOVERI RICHIESTI Al DIACONI}

D'altro canto, di questa pericope parenetica non affiora la preoccupazione d'indicare gli aspetti specifici del diaconato. È vero che questo brano illustra le qualità morali e i doveri richiesti ai diaconi. Ma è altrettanto vero che unanalisi di tali requisiti, porta a individuarvi soltanto dati piuttosto allusivi sullo specifico dell'identità e del ministero diaconali. Verrebbe da concludere che le qualità morali elencate qui per i diaconi dovrebbero essere coltivate dai cristiani in quanto tali.

Ad esempio, l'essere "degni di rispetto" è richiesto ai diaconi come al vescovo. Ma l'apostolo Paolo aveva già invitato ogni cristiano a pensare "cose dignitose" 43 . Similmente la moderazione nel bere vino è qui raccomandata ai diaconi, come al vescovo; ma nella Lettera agli Efesini l'esortazione è rivolta a tutti i fedeli ${ }^{44}$.

In questo elenco delle qualità morali e doveri richiesti ai diaconi, anche se non in modo esclusivo rispetto agli altri cristiani, l'unica eccezione è che essi dovevano essere sposati una volta sola. Perciò, alla morte della moglie, non era concessa loro la possibilità di nuove nozze ${ }^{45}$. Oppure - come sostengono alcuni studiosi contemporanei ${ }^{46}$ - questa prescrizione, fatta valere nelle lettere pastorali per i candidati sia al diaconato (1Tm $3,12)$ che all'episcopato $(3,2)$ e al presbiterato $(\mathrm{Tt} 1,6)$, sarebbe un cenno allobbligo della continenza dei diaconi, come dei vescovi e dei presbiteri. Comunque sia, che questa richiesta ai diaconi venga fatta immediatamente prima al vescovo e che nelle lettere pastorali venga ripetuta per i presbiteri, ma non per altri cristiani - ad eccezione della disposizione analoga prevista per le vedove (cf. 5,9) -, è un altro sintomo che i diaconi fossero intesi come figure ecclesiali sul versante del vescovo più che

${ }^{43}$ Cf. 1 Tm 3,8; Fil 4,8; Tt 2,2.

${ }^{44}$ Cf. 1 Tm 3,8; Tt 1,7; Ef 5,18.

${ }^{45}$ Cf. P. De Ambroggi, Le Epistole pastorali di S. Paolo a Timoteo e a Tito, Torino-Roma $1964^{2}$, p. 132.

${ }^{46}$ Cf. Ch. Cochini, Origines apostoliques du célibat sacerdotal, Genève 2006²; R. Cholij, Clerical Celibacy in East and West, Leominster 1988; A. M. Stickler, Il celibato ecclesiastico. La sua storia e i suoi fondamenti teologici, Città del Vaticano 1994; S. Heid, Zölibat in der frühen Kirche. Die Anfänge einer Enthalsamkeitspflicht für Kleriker in Ost und West, Schöningh-Paderborn 1997. 
su quello dei fedeli. Lo conferma anche l'aggiunta della richiesta sia ai diaconi che al vescovo di essere capaci di dirigere la propria famiglia e di educare i propri figli (3, 4 e v. 12). Ma forse è pure significativo che per i diaconi non sia esplicitato il motivo di questa richiesta, enunciato invece chiaramente per i vescovi: chi è capace di dirigere la propria famiglia, sarà anche in grado di prendersi cura della Chiesa (v. 5). Dunque, da un lato, in questa vicinanza dei diaconi al vescovo, che si prende cura dell'intera comunità cristiana, rintracciamo un elemento specifico del loro ministero. In effetti, la loro collaborazione subordinata al vescovo è diversa rispetto all'edificazione della Chiesa cui sono chiamati tutti gli altri cristiani. Dall'altro, sembra proprio che la Prima Lettera a Timoteo riservi la direzione - o presidenza - della comunità cristiana e, in specie, l'insegnamento pubblico al suo interno, direttamente al vescovo e ai presbiteri. Difatti, dal vescovo si esige che sia "capace d'insegnare" (didaktíkón, 3, 2) e che "sappia guidare bene - letteralmente presiedere (proïstámenon) - la propria famiglia [...] perché, se uno non sa guidare - presiedere (prostênaí) - la propria famiglia, come potrà aver cura della Chiesa di Dio?" $(3,4-5)$.

A proposito poi dei presbiteri, l'autore aggiunge in modo ancora più esplicito in 5,17 :

I presbiteri che esercitano bene la presidenza (proestôtes) siano considerati meritevoli di un duplice riconoscimento, soprattutto quelli che si affaticano nella predicazione e nell'insegnamento (en lógoi kaì didaskalíai).

I diaconi, invece, non sono chiamati a svolgere queste funzioni.

\section{I "SETTE" DEGLI ATTI DEGLI APOSTOLI}

\subsection{PROBLEMA ORGANIZZATIVO E TRADIZIONI SOCIO-RELIGIOSE DIFFERENTI}

Si deve rilevare che il brano di At 6,1-6, fin dai Padri della Chiesa, è stato tradizionalmente considerato come la testimonianza dell'istituzione del diaconato all'interno della Chiesa madre di Gerusalemme. Ciò nonostante, da tempo ormai, i biblisti contemporanei sono molto più cauti. 
Anzi, si è diffusa la tendenza a non considerare i "sette" come i primi diaconi della Chiesa gerosolimitana, benché studiosi, già negli anni cinquanta del secolo scorso, fossero giunti a supporre che i "sette" fossero i primi vescovi o presbiteri di Gerusalemme nominati dagli Apostoli ${ }^{47}$. Pur tenendo conto di prese di posizione del genere, inizierei a riprendere gli elementi salienti del racconto di Luca. Egli negli At 6,1 scrive:

In quei giorni, aumentando il numero dei discepoli, quelli di lingua greca mormorarono contro quelli di lingua ebraica perché, nellassistenza quotidiana, venivano trascurate le loro vedove.

In prima battuta, sembra che si trattasse di un problema piuttosto di carattere pratico: organizzare meglio la distribuzione degli alimenti destinati dalla comunità a un gruppo di vedove. In realtà, il problema organizzativo aveva radici molto più profonde. Difatti, Luca precisa che le vedove trascurate appartenevano alla cerchia dei cristiani detti "ellenisti" (tôn Hellēnistôn). In questo modo egli ci fa sapere che allinizio la Chiesa di Gerusalemme era bilingue: una parte di essa, di origine giudaica, parlava l'aramaico e leggeva la Bibbia ebraica; l'altra, invece, era costituita da cristiani che parlavano in greco e leggevano la Bibbia greca. Non erano di matrice pagana, ma provenivano dal giudaismo della diaspora e, per qualche ragione, erano tornati a vivere a Gerusalemme (cf. 6,9 $)^{48}$.

Dunque, pare che le vedove dei giudeo-cristiani "ellenisti" venissero dimenticate dalla comunità cristiana nella distribuzione quotidiana del cibo, a differenza delle vedove giudeo-cristiane di lingua ebraica, che lo ricevevano regolarmente. Di conseguenza, i cristiani "ellenisti” si misero a protestare contro quelli di lingua ebraica (pròs toùs Hebraíous), per la preferenza accordata alle vedove di questi ultimi.

In radice, stava accadendo che il gruppo di lingua ebraica, numericamente più consistente, continuava a osservare la legge mosaica e le tradizioni giudaiche. Invece, i cristiani di lingua greca erano probabilmen-

${ }^{47}$ Cf. P. Gaechter, Die Sieben. Apg, 6, 1-6, Zeitschrift für Katholische Theologie 74 (1952), p. 125-166.

${ }^{48}$ Cf. R. Pesch, "Hellenisten" und "Hebräer". Zu Apostelgeschichte 9, 29 und 6, 1, Biblische Zeitschrift 23 (1979), p. 87-92; G. Schneider, Die Apostelgeschichte, FreiburgBasel-Wien 1980, p. 406-416. 
te meno legati all'osservanza della legge. Del resto, fu proprio per questa ragione che, qualche anno dopo, la Chiesa avrebbe corso il rischio di un vero e proprio scisma tra le due componenti.

\subsection{IMPOSIZIONE DELLE MANI DEGLI APOSTOLI E AFFIDAMENTO DELLA DIACONIA}

Luca attesta che:

I Dodici convocarono il gruppo dei discepoli e dissero: "Non è giusto che noi lasciamo da parte la parola di Dio per servire alle mense (diakoneîn trapézais). Dunque, fratelli, cercate fra voi sette uomini di buona reputazione, pieni di Spirito e di sapienza, ai quali affideremo questo incarico. Noi, invece, ci dedicheremo alla preghiera e al servizio della Parola (têi diakoníai toû lógou)". Piacque questa proposta a tutto il gruppo e scelsero Stefano, uomo pieno di fede e di Spirito santo, Filippo, Pròcoro, Nicànore, Timone, Parmenàs e Nicola, un proselito di Antiochia. Lì presentarono agli apostoli e, dopo aver pregato, imposero loro le mani $(6,2-6)$.

Dato che la comunità cristiana ormai si stava ingrandendo e che la vita comunitaria stava diventando più complessa, i Dodici inventarono così una nuova struttura assistenziale, mirata a venire incontro in modo stabile ed efficace alla nuova esigenza comunitaria. Ma prima, ne avevano parlato con gli altri cristiani (cf. 6,2). Non avevano deciso da soli. Inoltre, dopo che "questa proposta era piaciuta a tutto il gruppo" $(6,5)$, avevano invitato i fedeli ad aiutarli anche nella scelta delle persone cui affidare il nuovo ministero ecclesiale $(6,3)$. Certo, i tre criteri evangelici della scelta dei sette - ossia la buona reputazione, la docilità allo Spirito Santo e la conseguente sapienza - erano stati decisi dagli apostoli. Tuttavia, era stata la comunità che, rispondendo volentieri alla richiesta degli apostoli, li aveva aiutati a individuare le persone adatte.

D’altro canto, erano stati gli apostoli a imporre le mani ai candidati ${ }^{49}$, con una preghiera (cf. 6,6) alla quale verosimilmente aveva preso parte la comunità. Dunque, è indubbio che a conferire ai "sette" questo ministero era stata l'incipiente gerarchia di cui la Chiesa si era dotata fin

49 Cf. C. M. Martini, Atti degli Apostoli, Cinisello Balsamo 19949 , p. 119. 
dalle origini. Come confermano soprattutto le lettere pastorali, l'imposizione delle mani era un segno rituale - con le nostre categorie potremmo dire un "segno sacramentale" - che trasmetteva un carisma, ossia un dono dello Spirito Santo, in vista dell'esercizio di una funzione ecclesiale ${ }^{50}$. Quel gesto degli apostoli esprimeva e attuava l'azione dello Spirito Santo che, attraverso di loro, chiamava i sette uomini per inviarli a esercitare un servizio ecclesiale ${ }^{51}$, orientato cioè all'edificazione della comunità cristiana e, in ultima analisi, alla salvezza dei fedeli.

Del resto, per alcuni biblisti, il giudizio sulla "buona reputazione" corrisponderebbe allesito della prova che, secondo la Prima Lettera a Timoteo $(3,10)$, era richiesta ai candidati al diaconato ${ }^{52}$.

\subsection{IL PROBLEMA DELL'IDENTITÀ DEl "SETTE"}

Evidenziata la nomina apostolica dell'incarico dei "sette", affrontiamo il problema centrale di questo brano, vale a dire: questi uomini sui quali gli apostoli, "dopo aver pregato, imposero le mani” per affidare loro il "servizio (diakonía) delle mense", possono essere considerati come "diaconi" in senso stretto?

In vista di una risposta, prendiamo le mosse dal livello terminologico. In questo brano ricorrono sia il sostantivo diakonía, per indicare il "servizio" della distribuzione quotidiana del cibo per le vedove bisognose, sia il verbo diakoneîn. Questo verbo però non designa solo il "servizio delle mense", ma anche il "servizio" della parola (têi diakonia tồ lógou, 6,4). Gli apostoli decidono di continuare a svolgere il ministero della predicazione all'interno della comunità cristiana, grazie allaiuto offerto loro dai sette uomini incaricati dell'altro ministero ecclesiale.

Resta vero che gli uomini su cui gli apostoli fanno il gesto rituale d'imporre le mani, non sono mai definiti diákonoi. Anzi, anche in At 21,8, Filippo "uno dei sette" (ek tôn heptá), non è chiamato "diacono". Tuttavia, abbiamo visto come, circa due decenni prima della stesura degli Atti, l'apostolo Paolo, nella Lettera ai Filippesi $(1,1)$, già menzionasse i "diaco-

${ }^{50}$ Cf. 1 Tm 4,14; $2 \operatorname{Tm} 1,6$.

${ }^{51}$ Cf. At 13,2.

${ }^{52}$ Cf. J. Lécuyer, Les diacres dans le Nouveau Testament, art. cit., p. 24. 
ni" subito dopo i "vescovi”. Perciò sarebbe molto improbabile che Luca, collaboratore di Paolo fin dal secondo viaggio missionario di questi, non fosse stato al corrente dellesistenza di "diaconi" nella Chiesa di Filippi, presso cui svolse per un certo tempo l'attività missionaria.

Ma se i "sette" che "servivano le mense" fossero stati intesi da Luca come diaconi, perché non lì ha definiti con il termine specifico, che peraltro sarebbe stato coerente con l'espressione diakoneîn trapézais, cioè "servire alle mense"? Si potrebbe rispondere che Luca proprio perché ha fatto ricorso al verbo diakonein, ha evitato di ripetere il sostantivo diákonos. D’altronde, Luca ha usato il sostantivo diakonía per designare al v. 2 il ministero dei sette nella distribuzione degli alimenti in modo identico a come l'ha ripetuto al v. 4 per indicare il ministero della predicazione ecclesiale degli apostoli. Comunque, pur ammettendo che i sette non fossero considerati da Luca come diaconi veri e propri, non pare affatto casuale questa sua insistenza sul campo semantico della diakonía in un brano incentrato sul ministero apostolico. Essa lascia trasparire un probabile intento di Luca: ricordare un aiuto concreto dato agli apostoli come esempio da proporre ai diaconi già attivi attorno agli anni Ottanta, quando egli stese gli Atti.

Ma potremmo spingerci ancora più in là: facendo memoria non di un servizio spontaneo di alcuni cristiani, ma dell'istituzione di un gruppo con finalità caritativa, di numero così preciso e simbolico, avvenuta mediante l'imposizione delle mani degli apostoli. Luca sembra mostrare ai suoi lettori come, fin dalle origini del cristianesimo, fosse sorta una necessità simile a quelle cui facevano fronte i diaconi della Chiesa dei suoi tempi ${ }^{53}$.

\subsection{CONFERME DEI PRIMI PADRI DELLA CHIESA}

Sta di fatto che l'attribuzione del titolo specifico di "diaconi" ai sette incaricati dagli apostoli non tarderà a venire. Ireneo di Lione nell'Adversus haereses ricorda che Stefano fu "eletto dagli apostoli il primo diacono

${ }^{53}$ Si tratta dell'interpretazione data ad At 6,1-6 da F. Roloff, Die Apostelgeschichte, Göttingen 1981, p. 108-109, che comunque esclude che Luca individui in questa vicenda "la fondazione del posteriore ufficio diaconale". 
(diákonos)" ${ }^{54}$. Inoltre, in una parte dello scritto giuntaci solo in latino, Ireneo, presentando il gruppo dei "nicolaiti", già bollati dall' Apocalisse di Giovanni (2,6), come eretici della Chiesa di Efeso, precisa che essi, avevano "per maestro Nicola, uno dei sette che per primi furono ordinati al diaconato (ad diaconium) dagli apostoli ${ }^{55}$.

Il Vescovo di Lione è una fonte non solo antichissima (l'Adversus haereses risale al 180 circa), ma anche molto attendibile, perché - come egli stesso racconta - da giovane era stato allievo dell'anziano Policarpo, vescovo di Smirne, a sua volta "discepolo degli apostoli" ${ }^{6}$. Per di più, l'attribuzione del titolo "diaconi" ai sette personaggi di At 6 è così indiscutibile per Ireneo, che non sente il bisogno di precisarla in alcun modo ${ }^{57}$.

Il carattere tradizionale di questa lettura di At 6 ha una conferma ancora più antica, benché non così esplicita, nella Lettera ai Trallensi $(2,3)$ d'Ignazio di Antiochia, scritta attorno all'anno 107, cioè circa settant’anni prima dell'Adversus haereses. Il vescovo di Antiochia, alludendo ad At 6, ritiene riduttivo intendere il ministero dei diaconi nel senso della distribuzione del cibo (cf. At 6,2) ${ }^{58}$. Con le sue parole:

È poi necessario che anche i diaconi (diakónous), che sono [al servizio] dei misteri di Gesù Cristo,

siano graditi a tutti in ogni modo. Infatti, non sono diaconi (diákonoi) di cibi e di bevande, ma servitori (hypérétai) della Chiesa di Dio ${ }^{59}$.

${ }^{54}$ Ireneo di Lione, Adversus haereses, III, 12,10, in: Irénée de Lyon. Contre les hérésies. Livre III. Tome II. Texte et traduction (Sources Chrétiennes), ed. A. Rousseau-L. Doutreleau, Paris 1974, p. 225.

${ }_{55}$ Id., Adversus haereses, I, 26,3, in: Irénée de Lyon. Contre les hérésies. Livre I. Tome II. Texte et traduction (Sources Chrétiennes), ed. A. Rousseau-L. Doutreleau, Paris 1979, p. 348.

${ }^{56}$ Id., Adversus haereses, III, 3,4, in: Irénée de Lyon. Contre les hérésies. Livre III. Tome II. Texte et traduction (Sources Chrétiennes), ed. A. Rousseau-L. Doutreleau, Paris 1979, p. 38-43.

${ }^{57}$ Cf. S. Zardoni, I diaconi nella chiesa. Ricerca storica e teologica sul diaconato, op. cit., p. 10 , nota 2 .

${ }^{58}$ Cf. ibidem.

59 Ignazio di Antiochia, Lettera ai Trallensi, 2, 3, in: Ignace d'Antioche - Polycarpe de Smyrne, Lettres. Martyre de Polycarpe. Texte grec, introduction, traduction et notes (Sources Chrétiennes), ed. P. Th. Camelot, Paris 1958, 112. 
Allora, è vero che l'indagine esegetica deve illustrare il dato neotestamentario. Ma è altrettanto vero che sarebbe piuttosto miope il biblista che, dichiarando di attenersi alle proprie competenze, non tenesse conto dell'esegesi di At 6 proposta da un testimone così vicino alle fonti neotestamentarie e così attento ai rapporti tra le figure dell'incipiente gerarchia ecclesiastica ${ }^{60}$.

Tutto sommato, se sta questa identificazione diaconale dei sette incaricati del servizio delle mense, possiamo rintracciare qualche indizio ulteriore sullo specifico del diaconato alla luce della testimonianza degli Atti su Stefano e su Filippo.

\section{IL SERVIZIO DELLA PAROLA DI STEFANO}

Per eleggere i sette uomini destinati alla diakonía delle mense, gli apostoli, oltre al criterio della buona reputazione, ne avevano individuati altri due, vale a dire: la pienezza di Spirito e la pienezza di sapienza (At $6,3)$. A proposito a Stefano, Luca tiene a precisare proprio che era un "uomo pieno di fede e di Spirito Santo" (6,5; cf. 7,55), per cui finí per contemplare Cristo glorioso e per imitarne il martirio. Ma prima ancora, grazie alla sua sapienza, frutto essa stessa dell'influsso dello Spirito, Stefano aveva la meglio nei contraddittori con i persecutori $(6,10)$. Si compiva in lui la promessa fatta dal Signore agli apostoli, che avrebbero avuto forza dal suo Spirito così da essergli testimoni ovunque $(1,8)$. Lo stesso Spirito Santo avrebbe parlato per mezzo di loro ${ }^{61}$. Difatti, Stefano, soprattutto con

${ }^{60}$ È noto che Ignazio di Antiochia è "il primo testimone della tradizione che distingue il ministero episcopale da quello presbiterale, altrimenti non deducibile direttamente dalla Scrittura" (cf. S. Zardoni, I diaconi nella chiesa. Ricerca storica e teologica sul diaconato, op. cit., p. 10, nota 2). Difatti, immediatamente dopo la frase citata, egli esorta tutti i cristiani a riverire "i diaconi (diakónous) come Gesù Cristo, come anche il vescovo (episkopon), che è l'immagine del Padre, e i presbiteri (presbytérous) come il sinedrio di Dio e l'assemblea degli apostoli: senza costoro la Chiesa non si può chiamare tale" (3, 1-2, in ibidem, p. 112). Cf. anche, R. Selejdak, Diakonat stały $w$ świetle dokumentów Soboru Watykańskiego II, posoborowego Urzędu Nauczycielskiego Kościoła i narodowych «Rationes institutionis diaconorum permanentium», Warszawa 2010; G. Schneider, Die Apostelgeschichte, op. cit., p. 417-419; R. Fabris, Atti degli Apostoli, Roma 1977, p. 201-206.

${ }^{61}$ Cf. Mt 10,19-20 (parallelo a Mc 13,11; a Lc 12,11-12 e anche a Lc 21,14-15); At 4,8 . 
i suoi discorsi, rese testimonianza a Cristo, fino a dare la vita per lui. La sintesi teologica della storia del popolo d'Israele da lui presentata davanti al sinedrio - sia pure riplasmata da Luca -, è un'attestazione vivida di come il suo servizio diaconale non si limitasse alla distribuzione di cibo, ma implicasse un'attività kerygmatica al di fuori della comunità cristiana.

\section{L'ATTIVITÀ MISSIONARIA DI FILIPPO}

Per quanto riguarda Filippo, un altro dei "sette" della Chiesa madre di Gerusalemme, gli Atti testimoniano che non solo annunciava la parola di Dio, ma svolgeva anche altre funzioni.

Quanto alla prima attività, è da sottolineare che Filippo fosse denominato "evangelista"62, non perché scrisse un Vangelo, ma perché annunciava l'“evangelo" - cioè la "bella notizia" - di Cristo. Prendeva parte così a un ministero ultimamente stabilito da Cristo stesso ${ }^{63}$ e attribuito dalla Seconda Lettera a Timoteo ${ }^{64}$ a un vescovo come Timoteo, benché Filippo non lo esercitasse in qualità di guida di una comunità cristiana.

Più precisamente, la sua azione missionaria in Samaria, in cui si era trasferito per sfuggire alla violenta persecuzione scoppiata a Gerusalemme (At 8,1-4), comprendeva tre tipi di funzioni: la predicazione della parola di Dio, incentrata su Cristo ${ }^{65}$; diverse attività caritative, come gli esorcismi e la cura dei malati $(8,7)$, fino alla loro guarigione miracolosa ${ }^{66}$; ma anche l'amministrazione del battesimo $(8,12-13)$.

A riguardo poi del battesimo, vale la pena osservare una seconda differenza ministeriale tra Filippo e gli apostoli. Filippo non imponeva le mani sui battezzati per donare loro lo Spirito Santo, come, poco dopo, si attesta degli apostoli Pietro e Giovanni (v. 17). Ma, al di là di questa differenza, Filippo battezzò (v. 38) il "funzionario di Candàce, regina di Etiopia" (v. 27), dopo avergli annunciato "la bella notizia di Gesù" (v. 35). Che non si trattasse di un fatto sporadico appare dalla conclusione

\footnotetext{
62 At 21,8: tồ euanggelistồ óntos.

${ }^{63}$ Cf. specialmente Ef 4,1.

${ }^{64}$ Cf. 2 Tm 4,5.

${ }^{65}$ Cf. At 8,4-5.12.

${ }^{66}$ Cf. ibidem, 8,6.
} 
del racconto, che ritrae Filippo come evangelizzatore in "tutte le città" da Azoto a Cesarea (v. 40). Ė più che verosimile che Luca, attestando che Filippo "evangelizzava", sintetizzi così una serie di attività kerygmatiche, battesimali e caritative, simili a quelle appena evocate in $8,5-7$, che Filippo avrebbe continuato a fare per anni.

In sintesi, nel ritratto di Filippo delineato negli Atti possiamo rintracciare una figura diaconale che svolgeva molteplici compiti di prima evangelizzazione: la predicazione dellevangelo di Cristo, lamministrazione del battesimo e varie forme di carità nei confronti di infermi, di posseduti dal demonio, nonché, sempre in vista della plantatio Ecclesiae, nei confronti di ospiti.

\section{CONCLUSIONE}

Basandoci sulle testimonianze dei Padri della Chiesa, soprattutto di quelle più antiche e affidabili ${ }^{67}$, si può dire che l'identificazione dei sette uomini di At 6 con delle figure diaconali costituisce linterpretazione del suddetto brano neotestamentario più che probabile. Tale interpretazione permette di determinare più precisamente qualche contenuto del ministero diaconale e di individuarne lorigine nella volontà d'istituzione degli apostoli. Stando così le cose, la prospettiva fondamentale del Nuovo Testamento è che nella Chiesa delle origini i diaconi siano sorti come aiuto degli apostoli e dei loro successori. In quel contesto era nitida la coscienza che gli stessi apostoli e i loro successori fossero servi di Cristo, il quale, a sua volta, aveva assunto per amore la "condizione del «servo" di Dio e degli uomini" ${ }^{68}$. Venuto non per essere servito, ma per servire, Cristo ha dato la vita per salvare tutti gli uomini. La diaconia è dunque la forma cristologica della Chiesa in quanto tale: per continuare ad essere strumento di salvezza nelle mani del Crocifisso risorto, la comunità cristiana e, al suo interno, ogni fedele, si lasciano docilmente "conformare" a Cristo-Servo dal suo stesso Spirito. In questorizzonte ecclesiale il diacono, in quanto cristiano, è icona vivente di Cristo-Servo nella Chiesa. Egli

${ }^{67}$ Alle testimonianze già citate vanno aggiunti anche i passi della Didachè 15,1-2 e di 1 Clemente 40,2-5; 42,4-5, 44,4.

68 Cf. Fil 2,6-7. 
è ordinatum ad ministerium, come lasciano già intravedere i cenni della Lettera ai Filippesi e della Prima Lettera a Timoteo. Più esattamente: i sette diaconi non si limitarono a svolgere funzioni caritative, come il "servizio delle mense". Gli Atti testimoniano che per lo meno Stefano e Filippo predicavano l'evangelo e che quest'ultimo amministrò anche il battesimo ${ }^{69}$. In questo senso - come dichiara la Lettera ai Trallensi $(2,3)$ di Ignazio di Antiochia -, i diaconi erano al servizio "dei misteri di Gesù Cristo", e non "di cibi e di bevande". Tutto sommato, la nostra indagine che ha tentato d'interpretare alcuni dati del Nuovo Testamento all'interno della vivente tradizione della Chiesa, mette in evidenza come la testimonianza neotestamentaria sulla figura e ministero dei diaconi consenta alla Chiesa contemporanea di attuare un discernimento sulloggi per continuare ad essere conforme a Cristo, Servo di Dio e dell'uomo.

Streszczenie. Figura i posługa diakona w perspektywie nowotestamentowej. Figura i posługa diakona są ukazane głównie w następujących fragmentach Nowego Testamentu: w Liście św. Pawła do Filipian 1, 1, jego Pierwszym Liście do Tymoteusza 3, 8-13 i Dziejach Apostolskich 6,1-6.8-15; 7; 8,4-13.25-40. List do Filipian (1,1), stanowiący najstarsze świadectwo, wspomina istnienie diakonów oraz ich współpracę z biskupami już we wspólnotach chrześcijańskich powstałych w czasach św. Pawła. Ponadto ich istnienie potwierdza Pierwszy List do Tymoteusza (3,8-13). Wskazane są w nim przymioty moralne, jakimi winni się odznaczać kandydaci do diakonatu, oraz wzmiankowany sposób ich przyjęcia do grona diakonów. Bardzo prawdopodobne ustanowienie diakonów przez apostołów wspominają natomiast Dzieje Apostolskie (6, 1-6). Ponadto Dzieje Apostolskie w kolejnych wersetach przywołują funkcje diakonów, które nie ograniczają się do realizacji działań charytatywnych, ale także, o czym świadczy historia Szczepana i Filipa, obejmują głoszenie ewangelii i udzielanie chrztu. W tym kontekście można powiedzieć, że teksty Nowego Testamentu przedstawiają diakonów jako tych, którzy dążą do coraz doskonalszego upodobnienia się do Chrystusa-Sługi, który mocą Ducha Świętego zachęca cały Kościół do bycia w większym stopniu ludem zdolnym do służenia drugiemu człowiekowi i do przywrócenia światu smaku służby.

Słowa kluczowe: Nowy Testament; Chrystus-Sługa; Duch Święty; apostołowie; biskupi; prezbiterzy; diakonia; diakoni; posługa diakońska; posługi kościelne; ewangelizacja; chrzest; „siedmiu”; przymioty moralne; funkcje diakonów. 
Abstract. The Figure and Ministry of the Deacon Within the Perspective of the New Testament. The figure and ministry of the deacon are presented mostly in the following fragments of the New Testament: in St. Paul's Letter to the Philippians (1, 1), in his First Letter to Timothy (3, 8-13) and in The Acts of the Apostles (6, 1-6.8-15; $7 ; 8,4-13.25-40)$. The oldest New Testament reference that we have is in the Letter to the Philippians, which recalls the existence of deacons in the Christian communities of Pauline origin, and these are presented as collaborators to the Bishops, to whom they are subordinate. Their existence is later confirmed in St. Paul's Letter to Timothy (3, 8-11), which lays out various conditions necessary for the admission of certain members of the faithful to the diaconate. The Institution of the diaconate by the Apostles however, is most likely originates in the text of The Acts of the Apostles $(6,1-6)$. Throughout this text, various references are made to the functions of deacons, which are not simply limited to charitable works, but as we see in the lives of Stephen and Philip, they also have the task of preaching the Gospel, with mention of Philip even administering baptism. In this context, we can say that the New Testament texts present deacons as those who seek to conform to Christ-the-Servant, by the power of the Holy Spirit, inviting the Church to be pre-eminently a people of service, so that the world may experience the taste of service.

Keywords: New Testament; Christ-the-Servant; Holy Spirit; Apostles; Bishops; Presbyters; Diaconía; Deacons; Diaconate Ministry; Ecclesial Ministries; Evangelisation; Baptism; “Thirst”; Moral Qualities; Diaconal Functions.

\section{BIBLIOGRAFIA}

Barbaglio G., Alla comunità di Filippi, in: Le lettere di Paolo, ed. G. Barbaglio-R. Fabris, Roma $1990^{2}$, p. 535-568.

Bellia G., Il diaconato permanente alle origini della Chiesa, Seminarium 48 (2008) 4, p. 449-686.

Beyer H. W., “diakonéo, diakonía, diákonos”, in: Theologisches Wörterbuch zum Neuen Testament, ed. G. Kittel-G.Friedrich, vol. II, Stuttgart 1935, col. 81-93.

Brandt W. H. G., Dienst und Dienen im Neuen Testament, Gütersloh 1931.

Cattaneo E., I ministeri nella Chiesa antica. Testi patristici dei primi tre secoli, Milano 1997. Cochini Ch., Origines apostoliques du célibat sacerdotal, Genève $2006^{2}$.

Cholij R., Clerical Celibacy in East and West, Leominster 1988.

Collins J. N., A Ministry for Tomorrow's Church, Journal of Ecumenical Studies 32 (1995), p. 159-178.

Collins J. N., Diakonía. Re-interpreting the Ancient Sources, New York - Oxford 1990.

Comissione Teologica Internazionale, Il diaconato: evoluzione e prospettive, La Civiltà Cattolica 154 (2003) 1, p. 253-335.

Castellucci E., Il "ministero della soglia". Una lettura teologica del diaconato a partire dal Nuovo Testamento, Seminarium 4 (2008), p. 615-647. 
De Ambroggi P., Le Epistole pastorali di S. Paolo a Timoteo e a Tito, Torino-Roma $1964^{2}$. Duncan G. S., St. Paul's Ephesian Ministry. A Reconstruction with Special Reference to the Ephesian Origin of the Imprisonment Epistles, London 1929.

Fabris R., Atti degli Apostoli, Roma 1977.

Fabris R., Lettera ai Filippesi. Struttura, commento e attualizzazione, Bologna 2001.

Gaechter P., Die Sieben. Apg 6, 1-6, Zeitschrift für Katholische Theologie 74 (1952), p. 125-166.

Georgi D., Die Gegner des Paulus im 2. Korintherbrief. Studien zur religiösen Propaganda in der Spätantike, Neukirchener-Vluyn 1964.

Gnilka J., Der Philipperbrief, Freiburg im Breisgau $1987^{4}$.

Koch G.-Langgärtner G.-Pompey H., Il diaconato nella Chiesa oggi. Saggio di un approfondimento teologico del diaconato, in: Il diaconato oggi, ed. autori vari, Roma 1970 , p. $55-63$.

Heid S., Zölibat in der frühen Kirche. Die Anfänge einer Enthalsamkeitspflicht für Kleriker in Ost und West, Schöningh-Paderborn 1997.

Ignazio di Antiochia, Lettera ai Trallensi, in: Ignace d'Antioche - Polycarpe de Smyrne, Lettres, Martyre de Polycarpe. Texte grec, introduction, traduction et notes (Sources Chrétiennes), ed. P. Th. Camelot, Paris 1958.

Ireneo di Lione, Adversus haereses, in: Irénée de Lyon. Contre les hérésies. Texte et traduction (Sources Chrétiennes), ed. A. Rousseau-L. Doutreleau, Paris 1974.

Lécuyer J., Les diacres dans le Nouveau Testament, in: Le diacre dans l'Église et le monde d'aujourd'hui, ed. P. Winninger-Y.Congar, Paris 1966, p. 15-26.

Martini C. M., Atti degli Apostoli, Cinisello-Balsamo $1994^{2}$.

Michaelis W., Die Gefangenschaft des Paulus in Ephesus und das Itinerar des Timotheus. Untersuchungen zur Chronologie des Paulus und der Paulusbriefe, Gütersloh 1925.

Penna R., La diakonía nelle Lettere ai Corinzi, in: Il diaconato permanente, ed. F. Marinelli, vol. II, Napoli 1983, p. 211-218.

Pesch R., "Hellenisten" und "Hebräer". Zu Apostelgeschichte 9.29 und 6, 1, Biblische Zeitschrift 23 (1979), p. 87-92.

Pikaza X., Il Vangelo di Marco, Roma 1996.

Roloff F., Die Apostelgeschichte, Göttingen 1981.

Schneider G., Die Apostelgeschichte, Freiburg-Basel-Wien 1980.

Selejdak R., Diakonat stały w świetle dokumentów Soboru Watykańskiego II, posoborowego Urzędu Nauczycielskiego Kościoła i narodowych «Rationes institutionis diaconorum permanentium», Warszawa 2010.

Stickler A. M., Il celibato ecclesiastico. La sua storia e i suoi fondamenti teologici, Città del Vaticano 1994.

Vorgrimler H., Per una teologia del diaconato, in: Il diaconato oggi, ed. autori vari, Roma 1970 , p. $45-53$.

Zardoni S., I diaconi nella Chiesa. Ricerca storica e teologica sul diaconato, Bologna 1983. 